\title{
Increased Risk of HIV-Associated Pulmonary Diseases by Environmental Factors
}

\section{Chinnapaiyan $\mathrm{S}^{*}$, Dutta R and Unwalla HJ}

Department of Immunology, Florida International University, USA

*Corresponding Author: Chinnapaiyan Srinivasan, Department of Immunology, Herbert Wertheim College of Medicine, Florida International University, 11200 SW 8th street, AHC-1 \# 421, Miami, FL33199, USA, Tel: 305-348-3442; Fax: 305-243-6992; E-mail: schinnap@fiu.edu

\section{Mini Review \\ Volume 3 Issue 1}

Received Date: June 22, 2018

Published Date: July 02, 2018

\section{Abstract}

People living with HIV (PLWH) are at increased risk of airway abnormalities and exacerbation of several lung diseases. Several diseases such as primary lung cancer, chronic obstructive pulmonary disease (COPD), asthma, pulmonary arterial hypertension, bronchial hyper responsiveness and sleep-disordered breathing can be exacerbated by environmental factors such as second-hand cigarette smoke, diesel exhaust, etc. In the United States, HIV smokers outnumber HIV nonsmokers by a 3-fold margin. Moreover, use tobacco is one of emerged as a leading cause of death and comorbidities in the highly active antiretroviral therapy (HAART) era. For pulmonary diseases in particularly, smoking is the most prevalent but preventable causes of morbidity and mortality affecting PLWH. Incidence of acute exacerbation of chronic obstructive pulmonary disease (AECOPD) is already elevated in HIV-infected compared to uninfected patients and current smoking and alcohol abuse further substantially increases the risk of AECOPD. Several studies have also reported a link between high exposure levels of air pollution and the development of airflow obstruction as well as prevalence of COPD. Some other environmental factors due to rapid urbanization and industrialization may also contribute to COPD in HIV, This is possibly due to increased occupational dust exposure. This review will discuss the current highly risk of HIVassociated pulmonary disease by environmental factors such as tobacco smoke and air pollution (includes outdoor and indoor air pollutant).

Keywords: HIV; Pulmonary disease; Cigarette smoke; Alcohol; Air pollution; COPD; Asthma; Chronic bronchitis

Abbreviations: PLWH: People living with HIV; COPD: Chronic Obstructive Pulmonary Disease; HAART: Highly Active Antiretroviral Therapy; AECOPD: Acute Exacerbation of Chronic Obstructive Pulmonary Disease;
PM: Particulate Matter; VOC: Volatile Organic Compounds; BHR: Bronchial Hyper Responsiveness; WHO: World Health Organization; CS: Cigarette Smoke; EPA: Environmental Protection Agency; MCC: Mucociliary 


\section{Open Access Journal of Pulmonary \& Respiratory Sciences}

clearance; CFTR: Cystic Fibrosis Transmembrane conductance Regulator.

\section{Introduction}

\section{Impact of Environmental Air Pollution Associated on Lung Diseases}

Environmental air pollution comprises pollutants directly emitted from construction sites, unpaved roads, smokestacks or fires. It also includes some other particles composed of highly reactive chemicals emitted from power plants, industries and automobiles. In indoor settings, Furthermore, it is a consequence of cooking, smoking, vacuuming and dusting. A number of reports have shown that exposure to fine particle pollutants causes respiratory problems such as asthma exacerbations, reduced lung development in children, and increased respiratory symptoms such as coughing, wheezing, shortness of breath, and lung cancer [1,2] Globally more than two million deaths occur every year as a direct consequence of air pollution through damage to the lungs and the respiratory system [3]. Around 2.1 and 0.47 million deaths are caused by fine particulate matter (PM) and ozone respectively [3,4]. In addition, biological substances such as allergens and microbial compounds are also considered hazardous pollutants because of their potential health hazards as PM. Inhaling particulate matter results in a number of health effects including exacerbation of chronic respiratory, cardiovascular diseases, decreased lung function which consequently results in increased hospital admissions, emergency room visits and premature mortality. In addition, mild problems associated with inhaling PM2.5 are found to include shortness of birth (dyspnea), chest discomfort and pain, coughing and wheezing [5-7]. The American Lung Association reported [8] that people with lung disease such as asthma, chronic pulmonary disease, chronic bronchitis and emphysema are at increased risk of morbidity and mortality due to PM.

\section{Impact of Outdoor Air Pollutants Associated with Lung Diseases}

The outdoor pollutants mainly constitute particulate matter (PM), ozone and sulfur dioxide. Automobile exhaust, fuel burning (including wood stoves and fireplaces), forest fires, industrial activity, construction, etc., constitutes the main source of PM. Ozone is primarily produced in the atmosphere when nitrogen oxides from vehicle exhauster acts with volatile organic compounds (VOC) chemically upon exposure to sunlight. Sulfur dioxide is produced from industrial sites such as smelters, paper mills, power plants and steel manufacturing plants
[9]. The major consequences of outdoor pollutants lead to several lung diseases including increased infant mortality, increased exacerbations in asthmatics, and hospitalization due to respiratory distress, clinic visits for lower respiratory tract diseases, and decreased lung growth and function.

\section{Impact of Indoor Air Pollutants Associated with Lung Diseases}

The major indoor air pollutants come from allergens (e.g. dust mites, animal allergens, cockroaches, moulds) and irritants (e.g. tobacco smoke, perfumes, cleaning agents, nitrogen oxides). In addition, coal, biomass fuel, carbon monoxide and second-hand smoke is a major sources of indoor air pollution. Allergens due to house dust mite allergens induce IgE-mediated sensitization which leads to the development of bronchial hyper responsiveness (BHR) and asthma [10]. Improper management of asthma can lead to emphysema thereby decreasing lung function [11]. Approximately 700 million of the world's children breathe air polluted air by tobacco smoke, particularly at home. The World Health Organization (WHO) considers the effects of urban outdoor and indoor air pollution as a critical public health problem, resulting in more than 2 million premature deaths every year [12].

\section{Risk of Tobacco Smoke Associated with Lung Diseases}

Tobacco smoke causes many diseases other than cancer in the lower respiratory tract affecting the bronchi and the alveoli. In addition, cigarette smoking promotes lung cancer, chronic bronchitis, emphysema as well as increases the risk of mortality from pneumonia. Cigarette smokers are at increased risk of pneumonia. In people living with HIV, lung infections are exacerbated due to smoking [13]. Many individual components of cigarette smoke (CS) such as nitrogen dioxide, carbon monoxide and various metals are considered toxic enough to be highly regulated by the environmental protection agency (EPA). CS is the most common causes of chronic bronchitis followed by long-term exposure to air pollution, dust and fumes from the environment. Moreover, cigarette smoke further enhances the inflammation of the bronchial tubes due to infections by viruses and bacteria. Chronic bronchitis often predisposes to pneumonia. Chronic inflammation due to inflammation is the primary cause of COPD, chronic airway obstruction, emphysema, leading to a progressive and irreversible decline of lung function [14]. Mucociliary clearance (MCC) is a play an important innate defense mechanism of the airways and protects against inhaled microbes, irritants 


\section{Open Access Journal of Pulmonary \& Respiratory Sciences}

and is essential for the clearance of respiratory pathogens. MCC is impaired in chronic airway diseases like COPD, chronic bronchitis and asthma and is particularly suppressed in smokers due to mucus hyper secretion and compromised ciliary beating. In people living with HIV MCC suppression, a consequence of HIV infection as well, is further exacerbated in by smoking tobacco or abusing illicit drugs. One of the principal targets of cigarette smoke in the airway is Cystic fibrosis transmembrane conductance regulator (CFTR). CFTR is suppressed by HIV proteins and this is exacerbated by smoke [15-19].

In our recent study, we demonstrate that CS potentiates HIV infection of bronchial epithelial cells by increased expression of canonical HIV receptors CD4 and chemokine receptor type 5 (CCR5)on bronchial epithelial cells. In addition to enhancing HIV infection of bronchial epithelial cells, CS individually and additively suppresses CFTR biogenesis and function, possibly explaining the increased incidence of lung infections in HIV patients and its exacerbation in HIV smokers [16, 17]. Diaz, et al. [20] reported that 114 HIV-infected persons compared with 44 age-, sex-, and smoking-matched HIV-uninfected control persons, $15 \%$ of the HIV-infected persons had emphysema on CT scan when compared with only $2 \%$ observed in non-infected people $(p=0.025)$. Likewise, another study demonstrated that HIV infection was associated with 50 to $60 \%$ increased odds of COPD diagnosis [21]. The findings by Bigna, et al. [22] suggest a high prevalence of COPD in the global population with HIV, COPD was significantly higher in HIV-positive patients than in HIV-negative controls, even after adjustment for tobacco consumption. In another study on people living with HIV in the US, smokers aged 40 years lose more than 6 years of life expectancy from smoking, possibly outweighing the loss from HIV infection itself [23]. CS is also hazardous in HIV-positive pregnant women in the US. Cigarette smoking is associated with a high increase in risk of pregnancy loss among HIVpositive than HIV-negative women even in the presence of potent highly active antiretroviral therapy [24].

\section{Conclusion}

Taken together, our review emphasizes that HIV acts as crucial risk factor in several pulmonary diseases and is associated with increased morbidity and mortality when exposed to environmental insults like tobacco smoke and air pollution (includes outdoor and indoor air pollutants). Additionally, tobacco smoking and other environmental insults constitute a significant risk of lung cancer development and deaths in people living with HIV. Other pulmonary diseases prevalent in people living with HIV namely chronic bronchitis, emphysema, asthma and pulmonary arterial hypertension are further exacerbated on account of tobacco smoke or environmental pollution. Health organizations across the world have to focus future research and attention on mitigating environmental pollution not only for people living with HIV but also for the general population on the whole. More resources will have to be devoted to address tobacco smoking or restricting smokers to specific areas to minimize exposure to second hand tobacco smoke. These steps will increase the life expectancy and quality of life of the global population as a whole and people living with HIV in particular. New therapeutic interventions will also need developing to reduce the morbidity and mortality due to HIV-associated pulmonary diseases in children and young adults.

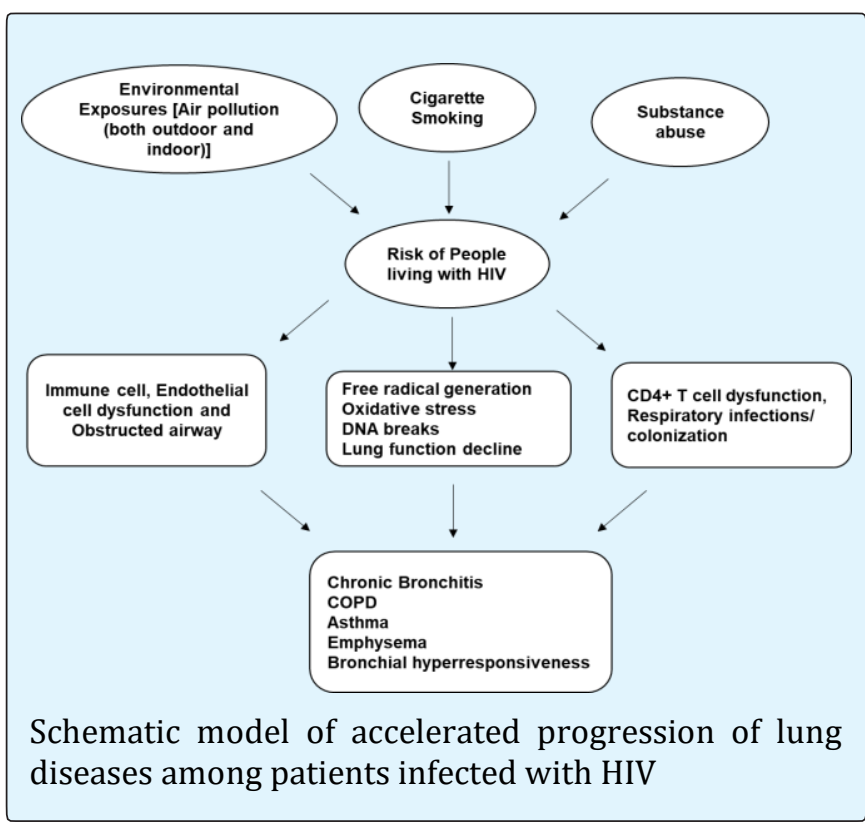

\section{Conflicts of Interest}

The authors report no conflicts of interest.

\section{References}

1. Mabahwi NAB, Leh OLH, Omar D (2014) Human Health and Wellbeing: Human Health Effect of Air Pollution. Procedia - Social and Behavioral Sciences 153(16): 221-229.

2. Kim KH, Kabir E, Kabir S (2015) A review on the human health impact of airborne particulate matter. Environment International 74: 136-143. 


\section{Open Access Journal of Pulmonary \& Respiratory Sciences}

3. Shah ASV et al. (2013) Global association of air pollution and heart failure: a systematic review and meta-analysis. The Lancet 382(9897): 1039-1048.

4. Chuang KJ, Yan YH, Chiu SY, Cheng TJ (2011) Longterm air pollution exposure and risk factors for cardiovascular diseases among the elderly in Taiwan. Occup Environ Med 68(1): 64-68.

5. Samoli E, Peng R, Ramsay T, Pipikou M, Touloumi G, et al. (2008) Acute effects of ambient particulate matter on mortality in Europe and North America: results from the APHENA study. Environ Health Perspect 116(11): 1480-1486.

6. Halonen JI, Lanki T, Yli-Tuomi T, Tiittanen P, Kulmala P, et al. (2009) Particulate air pollution and acute cardio respiratory hospital admissions and mortality among the elderly. Epidemiology 20(1): 143-153.

7. Guaita R, Pichiule M, Mate T, Linares C, Diaz J (2011) Short-term impact of particulate matter (PM(2.5)) on respiratory mortality in Madrid. Int J Environ Health Res 21(4): 260-274.

8. American Lung Association (2013). American Lung Association State of the Air 2013 - Health Effects of Ozone and Particle Pollution.

9. Golshan M, Faghihi M, Roushan- Zamir T, Masood Marandi M, Esteki B, et al. (2002) Early effects of burning rice farm residues on respiratory symptoms of villagers in suburbs of Isfahan, Iran. Int J Environ Health Res 12(2): 125-131.

10. Glasgow NJ, Ponsonby AL, Kemp A, Tovey E, Van Asperen P, et al. (2011) Feather bedding and childhood asthma associated with house dust mite sensitisation: a randomised controlled trial. Arch Dis Child 96(6): 541-547.

11. Chang C (2012) Asthma in children and adolescents: a comprehensive approach to diagnosis and management. Clin Rev Allergy Immunol 43(1-2): 98137.

12. World Health Organization (2006) WHO air quality guidelines for particulate matter, ozone, nitrogen dioxide and sulfur dioxide: Global assessment 2005: summary of risk assessment. Geneva, Switzerland: World Health Organization.

13. Rossouw TM, Anderson R, Feldman C (2015) Impact of HIV infection and smoking on lung immunity and related disorders. The European respiratory journal 46(6): 1781-1795.
14. Colarusso C, Terlizzi M, Molino A, Pinto A, Sorrentino $R$ (2017) Role of the inflammasome in chronic obstructive pulmonary disease (COPD). Oncotarget 8(47): 81813-81824.

15. Chinnapaiyan S, Unwalla HJ (2015) Mucociliary dysfunction in HIV and smoked substance abuse. Front Microbiol 6: 1052.

16. Chinnapaiyan S, Parira T, Dutta R, Agedelo M, Morris A, et al. (2017) HIV Infects Bronchial Epithelium and Suppresses Components of the Mucociliary Clearance Apparatus. PLoS One 12: e0169161.

17. Chinnapaiyan S, Dutta R, Bala J, Parira T, Agedelo M, et al. (2018) Cigarette smoke promotes HIV infection of primary bronchial epithelium and additively suppresses CFTR function. Sci Rep 8: 7984.

18. Unwalla H, Chinnapaiyan S, Periera T, Agudelo $M$, Morris A (2016) Effect of tobacco smoke and HIV infection on mucociliary clearance. Journal of Neuroimmune Pharmacology 11: S48-S48.

19. Unwalla HJ, Chinnapaiyan S, Dutta R, Nair M, Morris AM (2017) Cigarette Smoke Enhances Hiv-1 Infection of Nhbe Cells And Suppresses Cftr Function. American Journal of respiratory and critical care medicine 195: A5272.

20. Diaz PT, King ER, Wewers MD, Gadek JE, Neal D, et al. (2000) HIV infection increases susceptibility to smoking-induced emphysema. Chest 117 (5 Suppl): 285S

21. Crothers K, Butt AA, Gibert CL, Rodriguez-Barradas MC, Crystal S, et al. (2006) Increased COPD among HIV-positive compared to HIV-negative veterans. Chest 130(5): 1326-1333.

22. Bigna JJ, Kenne AM, Asangbeh SL, Sibetcheu AT (2018) Prevalence of chronic obstructive pulmonary disease in the global population with HIV: a systematic review and meta-analysis. Lancet Glob Health 6(2): e193-e202.

23. Reddy KP, Parker RA, Losina E, Baggett TP, Paltiel AD, et al. (2016) Impact of Cigarette Smoking and Smoking Cessation on Life Expectancy Among People With HIV: A US-Based Modeling Study. J Infect Dis 214(11): 1672-1681.

24. Westreich D, Cates J, Cohen M, Weber MK, Seidman D, et al. (2017) Smoking, HIV, and risk of pregnancy loss. AIDS 31: 553-560. 